Supporting Information

\title{
Comprehensive Lists of Internal Calibrants for Ultrahigh-Resolution Mass Spectrometry Analysis of Crude oil and Natural Organic Matter and their Preparation Recipes
}

\author{
Eunji $\mathrm{Cho}^{1}$, Nissa Nurfajrin Solihat ${ }^{2}$, Young Hwan $\mathrm{Kim}^{3,4 *}$, Sunghwan \\ $\operatorname{Kim}^{1,5,6^{*}}$ \\ 11 Department of Chemistry, Kyungpook National University, 80 Daehak-ro, Buk-gu, Daegu 41566, \\ Republic of Korea \\ ${ }^{2}$ Research Center for Biomaterials, Indonesian Institute of Sciences (LIPI), Cibinong 16911, Indonesia \\ ${ }^{3}$ Center for Research Equipment, Korea Basic Science Institute, 162 Yeongudanji-ro, Ochang-eup, \\ Cheongju-si, Chungcheongbuk-do 28119, Republic of Korea \\ ${ }^{4}$ Graduate School of Analytical Science and Technology, Chungnam National University, 99 Daehak-ro, \\ Yuseong-gu, Dae-jeon 34134, Republic of Korea \\ ${ }^{5}$ Department of Chemistry, Kyungpook National University, 80 Daehak-ro, Buk-gu, Daegu 41566, \\ Republic of Korea \\ ${ }^{6}$ Green-Nano Materials Research Center, Daegu 41566, Republic of Korea
}

\section{Corresponding author}

phone: 82-43-240-5140; fax: 82-42-865-3477;

e-mail: yhkim@kbsi.re.kr

phone: 82-53-950-5333; fax: 82-53-950-6330;

e-mail: sunghwank@knu.ac.kr 


\section{$<$ Table of Contents >}

Page

Table S1. Properties of samples used in this study.

Table S2. Experimental parameters for each ionization method used in this study. S-4

Table S3. List of standard compounds used in this study.

S-5

Table S4. Calibration peak lists for a) APPI and APCI, b) NaTFA solution, c)

S-6 arginine solution for ESI and LDI, and d) RP solution for LDI analysis.

Figure S1. Comparison of broadband spectra obtained upon varying the mixing S-8 ratio of the ESA crude oil and calibration solution from a) (+) APPI FT-ICR MS, b) (+) APCI FT-ICR MS, and c) (-) APCI FT-ICR MS.

Figure S2. Broadband spectra and mass accuracy plots for the EOC crude oil mixed with calibration solution obtained with a) (+) APPI, b) (+) APCI, and c) (-) APCI.

Figure S3. Comparison of broadband spectra obtained by varying the mixing ratio of the ESA crude oil and NaTFA solution from a) (+) ESI FT-ICR MS and b) (-) ESI FT-ICR MS.

Figure S4. Broadband spectra and mass accuracy plots for the EOC crude oil mixed with NaTFA solution obtained using a) (+) ESI and b) (-) ESI.

Figure S5. Comparison of broadband spectra obtained by varying the mixing ratio S-14 of the SRHA and a) NaTFA or b) arginine solutions obtained using (-) ESI FT-ICR MS.

Figure S6. Comparison of broadband spectra obtained upon varying the ratio of S-16 the NAP crude oil and RP solution obtained by a) (+) LDI FT-ICR MS and b) (-) LDI FT-ICR MS.

Figure S7. Broadband spectra and mass accuracy plots for the QHD crude oil mixed with RP solution obtained by a) (+) LDI and b) (-) LDI.

Figure S8. Comparison of broadband spectra obtained by varying the mixing ratio S-19 of the SRFA and a) NaTFA or b) RP solutions using (-) LDI FT-ICR MS.

Figure S9. Comparison of broadband spectra obtained by varying the mixing ratio of the SRHA and a) NaTFA or b) RP solutions using (-) LDI FT-ICR MS.

Figure S10. Broadband spectra for the ES mixed with a) arginine solution, b) NaTFA solution, or c) RP solution obtained using (-) LDI FT-ICR MS. 
Table S1. Properties of samples used in this study.

\begin{tabular}{|c|c|c|c|c|c|c|c|c|c|c|}
\hline Name & $\begin{array}{c}\mathrm{S} \\
\mathrm{wt} \%\end{array}$ & $\begin{array}{c}\mathrm{N} \\
\mathrm{ppm}\end{array}$ & TAN & API & $\begin{array}{c}\mathrm{Fe} \\
\mathrm{ppm}\end{array}$ & $\begin{array}{c}\mathrm{V} \\
\mathrm{ppm}\end{array}$ & $\begin{array}{c}\mathrm{Ni} \\
\mathrm{ppm}\end{array}$ & \multicolumn{3}{|c|}{ Origin } \\
\hline $\begin{array}{c}\text { EOC } \\
\text { crude oil }\end{array}$ & 4.79 & 2136 & 0.27 & 18.3 & 2.3 & 54.6 & 21.4 & \multicolumn{3}{|c|}{ Partitioned Zone } \\
\hline $\begin{array}{c}\text { ESA } \\
\text { crude oil }\end{array}$ & 0.18 & 3532 & 0.79 & 23.1 & 11.5 & 3.7 & 3.9 & \multicolumn{3}{|c|}{ Argenttina } \\
\hline $\begin{array}{l}\text { QHD } \\
\text { crude oil }\end{array}$ & 0.25 & 4405 & 3.15 & 16.1 & - & - & - & \multicolumn{3}{|c|}{ China } \\
\hline $\begin{array}{c}\text { NAP } \\
\text { crude oil }\end{array}$ & 2.01 & 4011 & 0.18 & 19.5 & - & 329.8 & 129.0 & \multicolumn{3}{|c|}{ Ecuador } \\
\hline Name & $\begin{array}{l}\mathrm{H}_{2} \mathrm{O} \\
\mathrm{wt} \%\end{array}$ & $\begin{array}{l}\text { ASH } \\
\text { wt } \%\end{array}$ & $\begin{array}{c}\mathrm{C} \\
\mathrm{wt} \%\end{array}$ & $\begin{array}{c}\mathrm{H} \\
\mathrm{wt} \%\end{array}$ & $\begin{array}{c}\mathrm{O} \\
\mathrm{wt} \%\end{array}$ & $\begin{array}{c}\mathrm{N} \\
\mathrm{wt} \%\end{array}$ & $\begin{array}{c}\mathrm{S} \\
\mathrm{wt} \%\end{array}$ & $\begin{array}{c}\mathrm{P} \\
\mathrm{wt} \%\end{array}$ & $\begin{array}{l}{ }^{13} \mathrm{C} \\
\text { wt } \%\end{array}$ & $\begin{array}{l}{ }^{15} \mathrm{~N} \\
\mathrm{wt} \%\end{array}$ \\
\hline SRHA & 1.52 & ND & 2.9 & ND & ND & 0.25 & ND & ND & ND & ND \\
\hline SRFA & 11.3 & 0.78 & 53.3 & 3.98 & $\begin{array}{c}41.8 \\
1\end{array}$ & 0.66 & 0.41 & ND & ND & ND \\
\hline $\begin{array}{c}\text { Elliott } \\
\text { STD soil }\end{array}$ & 1.52 & ND & 2.8 & ND & ND & 0.25 & ND & ND & ND & ND \\
\hline
\end{tabular}


Table S2. Experimental parameters for each ionization methods used in this study.

\begin{tabular}{|c|c|c|c|c|c|c|c|c|c|}
\hline & \multicolumn{7}{|c|}{ Crude oil } & \multicolumn{2}{|c|}{$\mathrm{NOM}$} \\
\hline Parameter, unit & $+\mathrm{ESI}$ & -ESI & $+\mathrm{APPI}$ & $+\mathrm{APCI}$ & -APCI & $+\mathrm{LDI}$ & -LDI & -ESI & -LDI \\
\hline Capillary exit, V & 210 & -220 & 210 & 220 & -220 & 210 & -220 & -220 & -150 \\
\hline Deflector plate, $\mathrm{V}$ & 200 & -200 & 200 & 200 & -200 & 200 & -200 & -200 & -200 \\
\hline Funnel 1, V & 150 & -150 & 150 & 150 & -150 & 150 & -150 & -150 & -150 \\
\hline Skimmer 1, V & 15 & -45 & 15 & 30 & -30 & 15 & -45 & -100 & -45 \\
\hline Funnel RF Amplitude, V & 130 & 120 & 110 & 100 & 110 & 90 & 100 & 120 & 100 \\
\hline Q1 mass, m/z & 200 & 200 & 200 & 180 & 180 & 200 & 200 & 200 & 150 \\
\hline Transfer Exit Lens, V & -20 & 20 & -20 & -20 & 20 & -20 & 20 & 20 & 20 \\
\hline Analyzer Entrance, V & -2.0 & 2.0 & -2.0 & -9.5 & 10 & -2.0 & 2.0 & 2.0 & 2.0 \\
\hline Side Kick, V & 0.0 & 0.5 & 0.0 & 0.0 & 0.0 & 0.0 & 0.5 & 0.5 & 0.5 \\
\hline Side Kick Offset, V & -1.0 & 1.0 & -1.0 & -1.5 & 0.5 & -1.0 & 1.0 & 1.0 & 1.0 \\
\hline Front Trap Plate, V & 1.5 & -1.6 & 1.5 & 1.5 & -1.5 & 1.5 & -1.6 & -1.6 & -1.6 \\
\hline Back Trap plate, V & 1.5 & -1.6 & 1.5 & 1.5 & -1.5 & 1.5 & -1.6 & -1.6 & -1.6 \\
\hline Back Trap plate Quench, V & -30 & 30 & -30 & -30 & 30 & -30 & 30 & 30 & 30 \\
\hline Scans & 100 & 100 & 100 & 100 & 100 & 200 & 200 & 300 & 200 \\
\hline Ion accumulation time, sec & 0.01 & 0.05 & 0.03 & 0.03 & 0.5 & 0.05 & 0.05 & 0.05 & 0.05 \\
\hline Flowrate, $\mu \ell /$ hour & 180 & 180 & 180 & 500 & 600 & - & - & 180 & - \\
\hline Nebulizer, bar & 0.8 & 0.8 & 1.5 & 2.0 & 2.0 & - & - & 0.8 & - \\
\hline Nebulizer temperature, ${ }^{\circ} \mathrm{C}$ & 400 & 400 & 300 & 350 & 370 & - & - & - & - \\
\hline Dry gas, $\mathrm{L} / \mathrm{min}$ & 4.0 & 4.0 & 4.0 & 3.5 & 4.0 & - & - & 4.0 & - \\
\hline Dry temperature, ${ }^{\circ} \mathrm{C}$ & 300 & 300 & 250 & 250 & 220 & - & - & 200 & - \\
\hline Grid increment & - & - & - & - & - & 1 & 1 & - & 1 \\
\hline Grid offset & - & - & - & - & - & 1 & 1 & - & 1 \\
\hline Laser focus & - & - & - & - & - & small & small & - & small \\
\hline Smart walk grid Width, $\mu \mathrm{m}$ & - & - & - & - & - & 2000 & 500 & - & 500 \\
\hline Laser power & - & - & - & - & - & 35 & 35 & - & 40 \\
\hline Laser shots & - & - & - & - & - & 300 & 300 & - & 30 \\
\hline Frequency, $\mathrm{Hz}$ & - & - & - & - & - & 1000 & 500 & - & 500 \\
\hline
\end{tabular}


Table S3. List of standard compounds used in this study.

\begin{tabular}{|c|c|c|c|c|}
\hline & Name & $\begin{array}{l}\text { Molecular } \\
\text { formula }\end{array}$ & $\begin{array}{c}\text { Molecular } \\
\text { weight } \\
(\mathrm{g} / \mathrm{mol})\end{array}$ & Sturucture \\
\hline \multirow{5}{*}{$\begin{array}{l}\text { APPI/APCI } \\
\text { Positive }\end{array}$} & 9-Phenyl anthracene & $\mathrm{C}_{20} \mathrm{H}_{14}$ & 254.33 & \\
\hline & Coronene & $\mathrm{C}_{24} \mathrm{H}_{12}$ & 300.35 & \\
\hline & $\begin{array}{l}\text { 9,10-Di(1-naphthyl) } \\
\text { anthracene }\end{array}$ & $\mathrm{C}_{34} \mathrm{H}_{22}$ & 430.55 & \\
\hline & Rubrene & $\mathrm{C}_{42} \mathrm{H}_{28}$ & 532.67 & \\
\hline & \multicolumn{4}{|c|}{$\begin{array}{l}<\text { Standard mixture preparation }> \\
\text { Mix } 10 \mu \mathrm{M} \text { 9-Phenyl anthracene } 100 \mu \ell, 10 \mu \mathrm{M} \text { Coronene } 200 \mu \ell, 1 \mu \mathrm{M} \\
9,10-\operatorname{Di}(1 \text {-naphthyl)anthracene } 150 \mu \ell, 1 \mu \mathrm{M} \text { Rubrene } 150 \mu \ell \text {, and } \\
\text { Tuningmix } 100 \mu \ell \text { to final concentrations of 9-Phenyl anthracene } 1.43 \\
\mu \mathrm{M} \text {, Coronene } 2.86 \mu \mathrm{M}, 9,10-\mathrm{Di}(1 \text {-naphthyl)anthracene } 0.21 \mu \mathrm{M} \text {, and } \\
\text { Rubrene } 0.21 \mu \mathrm{M} \text {. } \\
\text { All standards except the tuning mix are diluted with toluene. }\end{array}$} \\
\hline \multirow{4}{*}{$\begin{array}{c}\text { APCI } \\
\text { Negative }\end{array}$} & $\begin{array}{l}\text { 9-Anthracenecarboxylic } \\
\text { acid }\end{array}$ & $\mathrm{C}_{15} \mathrm{H}_{10} \mathrm{O}_{2}$ & 222.24 & \\
\hline & Heptadecanoic acid & $\mathrm{C}_{17} \mathrm{H}_{34} \mathrm{O}_{2}$ & 270.45 & \\
\hline & Stearic acid & $\mathrm{C}_{18} \mathrm{H}_{36} \mathrm{O}_{2}$ & 284.48 & \\
\hline & \multicolumn{4}{|c|}{$\begin{array}{l}<\text { Standard mixture preparation }> \\
\text { Mix } 10 \mu \mathrm{M} 9 \text {-Anthracenecarboxylic acid } 150 \mu \ell, 10 \mu \mathrm{M} \text { Heptadecanoic } \\
\text { acid } 60 \mu \ell, 10 \mu \mathrm{M} \text { Stearic acid } 120 \mu \ell \text {, and Tuning mix } 80 \mu \ell \text { to final } \\
\text { concentrations of 9-Anthracenecarboxylic acid } 3.66 \mu \mathrm{M} \text {, Heptadecanoic } \\
\text { acid } 1.46 \mu \mathrm{M} \text {, and Stearic acid } 1.95 \mu \mathrm{M} \text {. } \\
\text { All standards except the Tuning mix are diluted with methanol. }\end{array}$} \\
\hline
\end{tabular}


Table S4. Calibration peak lists for a) APPI and APCI, b) NaTFA solution, c) arginine solution for ESI and LDI, and d) RP solution for LDI analysis.

a) 4STDs+APCI-L Tuning mix (+) mode

\begin{tabular}{|c|c|c|c|c|c|}
\hline $\begin{array}{c}\text { Molecular } \\
\text { formula }\end{array}$ & $m / z$ & $\begin{array}{c}\text { Final } \\
\text { concentrati } \\
\text { on }\end{array}$ & $\begin{array}{c}\text { Molecular } \\
\text { formula }\end{array}$ & $\mathrm{m} / z$ & $\begin{array}{c}\text { Final } \\
\text { concentrati } \\
\text { on }\end{array}$ \\
\hline$\left[\mathrm{C}_{20} \mathrm{H}_{14}\right]^{+\bullet}$ & 254.10900 & $1.43 \mu \mathrm{M}$ & {$\left[\mathrm{C}_{15} \mathrm{H}_{10} \mathrm{O}_{2}-\mathrm{H}\right]^{-}$} & 221.06080 & $3.66 \mu \mathrm{M}$ \\
\hline$\left[\mathrm{C}_{24} \mathrm{H}_{12}\right]^{+\bullet}$ & 300.09335 & $2.86 \mu \mathrm{M}$ & {$\left[\mathrm{C}_{17} \mathrm{H}_{34} \mathrm{O}_{2}-\mathrm{H}\right]^{-}$} & 269.24860 & $1.46 \mu \mathrm{M}$ \\
\hline$\left[\mathrm{C}_{34} \mathrm{H}_{22}\right]^{+\bullet}$ & 430.17160 & $0.21 \mu \mathrm{M}$ & {$\left[\mathrm{C}_{18} \mathrm{H}_{36} \mathrm{O}_{2}-\mathrm{H}\right]^{-}$} & 283.26425 & $1.95 \mu \mathrm{M}$ \\
\hline$\left[\mathrm{C}_{42} \mathrm{H}_{28}\right]^{+\cdot}$ & 532.21855 & $0.21 \mu \mathrm{M}$ & $\begin{array}{c}{\left[\mathrm{C}_{10} \mathrm{H}_{16} \mathrm{O}_{6} \mathrm{~N}_{3} \mathrm{P}_{3}\right.} \\
\mathrm{F}_{10}-\mathrm{H}^{-}\end{array}$ & 556.00195 & - \\
\hline$\left[\mathrm{C}_{12} \mathrm{H}_{19} \mathrm{O}_{6} \mathrm{~N}\right.$ & 622.02896 & - & $\begin{array}{c}{\left[\mathrm{C}_{15} \mathrm{H}_{16} \mathrm{O}_{6} \mathrm{~N}_{3} \mathrm{P}_{3}\right.} \\
\left.\mathrm{F}_{3} \mathrm{~F}_{12}\right]\end{array}-\mathrm{H}^{-}$ & 805.98598 & - \\
\hline $\begin{array}{c}\mathrm{C}_{18} \mathrm{H}_{19} \mathrm{O}_{6} \mathrm{~N}_{3} \\
\mathrm{P}_{3} \mathrm{~F}_{24}\end{array}$ & 922.00980 & - & $\begin{array}{c}{\left[\mathrm{C}_{20} \mathrm{H}_{16} \mathrm{O}_{6} \mathrm{~N}_{3} \mathrm{P}_{3}\right.} \\
\mathrm{F}_{30}-\mathrm{H}^{-}\end{array}$ & 1055.97002 & - \\
\hline $\begin{array}{c}\mathrm{C}_{26} \mathrm{H}_{19} \mathrm{O}_{6} \mathrm{~N}_{3} \\
\mathrm{P}_{3} \mathrm{~F}_{40}\end{array}$ & 1321.98425 & - & & & \\
\hline
\end{tabular}

b)

\begin{tabular}{|c|c|c|c|}
\hline \multicolumn{2}{|c|}{ NaTFA (+) mode } & \multicolumn{2}{c|}{ NaTFA (-) mode } \\
\hline Molecular formula & $m / z$ & Molecular formula & $m / z$ \\
\hline$\left[\mathrm{Na}_{3}\left(\mathrm{CF}_{3} \mathrm{CO}_{2}\right)_{2}\right]^{+}$ & 294.93884 & {$\left[\mathrm{Na}\left(\mathrm{CF}_{3} \mathrm{CO}_{2}\right)_{2}\right]^{-}$} & 248.96040 \\
\hline$\left[\mathrm{Na}_{4}\left(\mathrm{CF}_{3} \mathrm{CO}_{2}\right)_{3}\right]^{+}$ & 430.91365 & {$\left[\mathrm{Na}_{2}\left(\mathrm{CF}_{3} \mathrm{CO}_{2}\right)_{3}\right]^{-}$} & 384.93520 \\
\hline$\left[\mathrm{Na}_{5}\left(\mathrm{CF}_{3} \mathrm{CO}_{2}\right)_{4}\right]^{+}$ & 566.88845 & {$\left[\mathrm{Na}_{3}\left(\mathrm{CF}_{3} \mathrm{CO}_{2}\right)_{4}\right]^{-}$} & 520.91001 \\
\hline$\left[\mathrm{Na}_{6}\left(\mathrm{CF}_{3} \mathrm{CO}_{2}\right)_{5}\right]^{+}$ & 702.86326 & {$\left[\mathrm{Na}_{4}\left(\mathrm{CF}_{3} \mathrm{CO}_{2}\right)_{5}\right]^{-}$} & 656.88482 \\
\hline$\left[\mathrm{Na}_{7}\left(\mathrm{CF}_{3} \mathrm{CO}_{2}\right)_{6}\right]^{+}$ & 838.83807 & {$\left[\mathrm{Na}_{5}\left(\mathrm{CF}_{3} \mathrm{CO}_{2}\right)_{6}\right]^{-}$} & 792.85963 \\
\hline$\left[\mathrm{Na}_{8}\left(\mathrm{CF}_{3} \mathrm{CO}_{2}\right)_{7}\right]^{+}$ & 974.81288 & {$\left[\mathrm{Na}_{6}\left(\mathrm{CF}_{3} \mathrm{CO}_{2}\right)_{7}\right]^{-}$} & 928.83444 \\
\hline
\end{tabular}


Table S4. Calibration peak lists for a) APPI and APCI, b) NaTFA solution, c) arginine solution for ESI and LDI, and d) RP solution for LDI analysis.

c)

\begin{tabular}{|c|c|}
\hline \multicolumn{2}{|c|}{ Arginine (-) mode } \\
\hline Molecular formula & $\mathrm{m} / \mathrm{z}$ \\
\hline$[(\mathrm{Arg})-\mathrm{H}]^{-}$ & 173.10440 \\
\hline$\left[(\mathrm{Arg})_{2}-\mathrm{H}\right]^{-}$ & 347.21607 \\
\hline$\left[(\mathrm{Arg})_{3}-\mathrm{H}\right]^{-}$ & 521.32775 \\
\hline$\left[(\mathrm{Arg})_{4}-\mathrm{H}\right]^{-}$ & 695.43943 \\
\hline$\left[(\mathrm{Arg})_{5}-\mathrm{H}\right]^{-}$ & 869.5510 \\
\hline
\end{tabular}

d)

\begin{tabular}{|c|c|c|c|}
\hline \multicolumn{2}{|c|}{ Phosphorus, red (+) mode } & \multicolumn{2}{c|}{ Phosphorus, red (-) mode } \\
\hline Molecular formula & $\mathrm{m} / z$ & Molecular formula & $\mathrm{m} / z$ \\
\hline$\left[\mathrm{P}_{7}\right]^{+}$ & 216.81578 & {$\left[\mathrm{P}_{7}\right]^{-}$} & 216.81688 \\
\hline$\left[\mathrm{P}_{9}\right]^{+}$ & 278.76331 & {$\left[\mathrm{P}_{9}\right]^{-}$} & 278.76440 \\
\hline$\left[\mathrm{P}_{11}\right]^{+}$ & 340.71083 & {$\left[\mathrm{P}_{11}\right]^{-}$} & 340.71193 \\
\hline$\left[\mathrm{P}_{13}\right]^{+}$ & 402.65835 & {$\left[\mathrm{P}_{13}\right]^{-}$} & 402.65945 \\
\hline$\left[\mathrm{P}_{15}\right]^{+}$ & 464.60587 & {$\left[\mathrm{P}_{15}\right]^{-}$} & 464.60697 \\
\hline$\left[\mathrm{P}_{17}\right]^{+}$ & 526.55340 & {$\left[\mathrm{P}_{17}\right]^{-}$} & 526.55449 \\
\hline$\left[\mathrm{P}_{19}\right]^{+}$ & 588.50092 & {$\left[\mathrm{P}_{19}\right]^{-}$} & 588.50202 \\
\hline$\left[\mathrm{P}_{21}\right]^{+}$ & 650.44844 & {$\left[\mathrm{P}_{21}\right]^{-}$} & 650.44954 \\
\hline$\left[\mathrm{P}_{23}\right]^{+}$ & 712.39597 & {$\left[\mathrm{P}_{23}\right]^{-}$} & 712.39706 \\
\hline$\left[\mathrm{P}_{25}\right]^{+}$ & 774.34349 & {$\left[\mathrm{P}_{25}\right]^{-}$} & 774.34459 \\
\hline$\left[\mathrm{P}_{27}\right]^{+}$ & 836.29101 & {$\left[\mathrm{P}_{27}\right]^{-}$} & 836.29211 \\
\hline$\left[\mathrm{P}_{29}\right]^{+}$ & 898.23854 & {$\left[\mathrm{P}_{29}\right]^{-}$} & 898.23963 \\
\hline$\left[\mathrm{P}_{31}\right]^{+}$ & 960.18606 & {$\left[\mathrm{P}_{31}\right]^{-}$} & 960.18716 \\
\hline
\end{tabular}


Figure S1. Comparison of broadband spectra obtained upon varying the mixing ratio of the ESA crude oil and calibration solution from a) (+) APPI FT-ICR MS, b) (+) APCI FT-ICR MS, and c) (-) APCI FT-ICR MS.
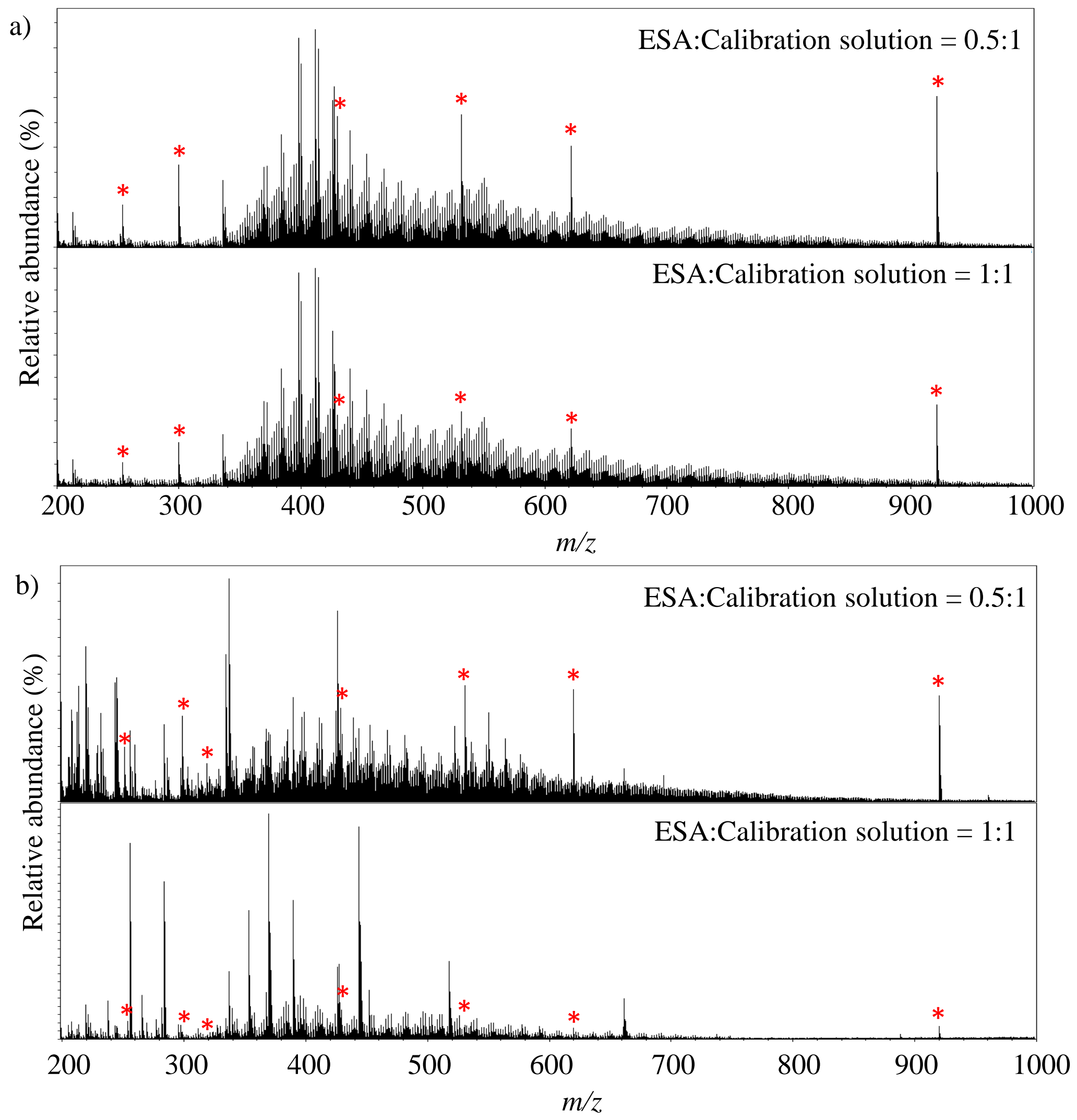
Figure S1. Comparison of broadband spectra obtained upon varying the mixing ratio of the ESA crude oil and calibration solution from a) (+) APPI FT-ICR MS, b) (+) APCI FT-ICR MS, and c) (-) APCI FT-ICR MS.

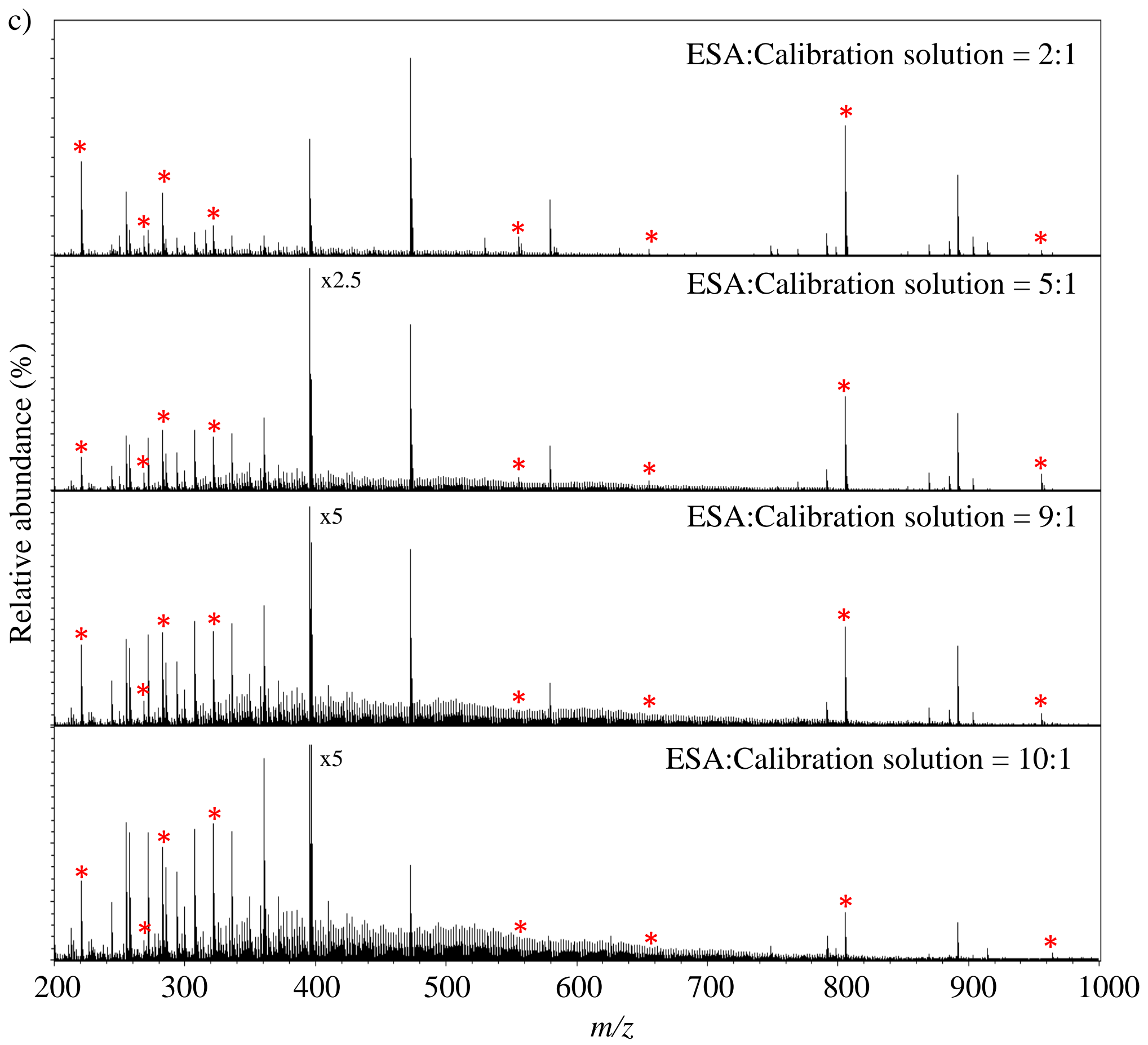


Figure S2. Broadband spectra and mass accuracy plots for the EOC crude oil mixed with calibration solution obtained with a) (+) APPI, b) (+) APCI, and c) (-) APCI.

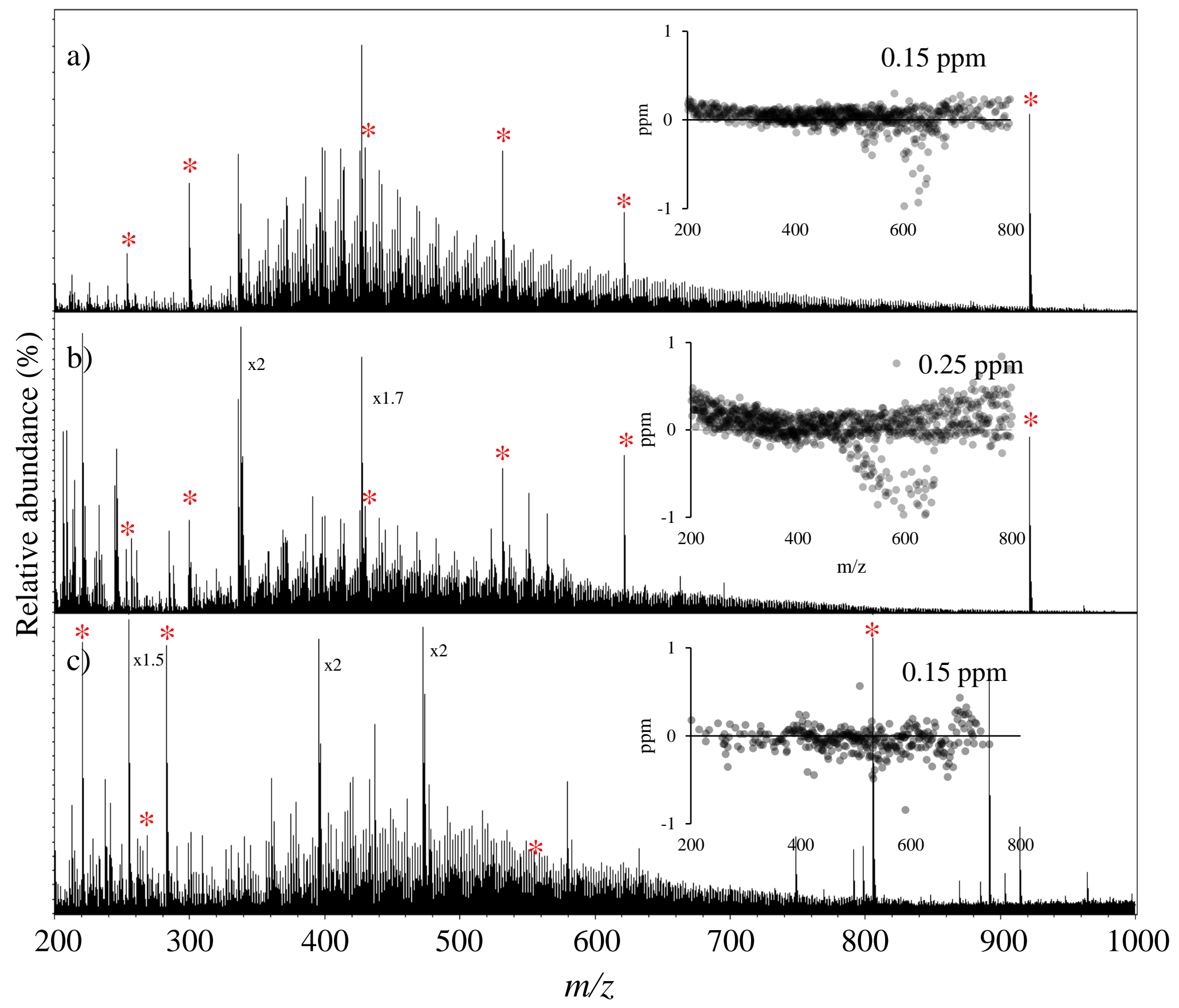


Figure S3. Comparison of broadband spectra obtained by varying the mixing ratio of the ESA crude oil and NaTFA solution from a) (+) ESI FT-ICR MS and b) (-) ESI FT-ICR MS.

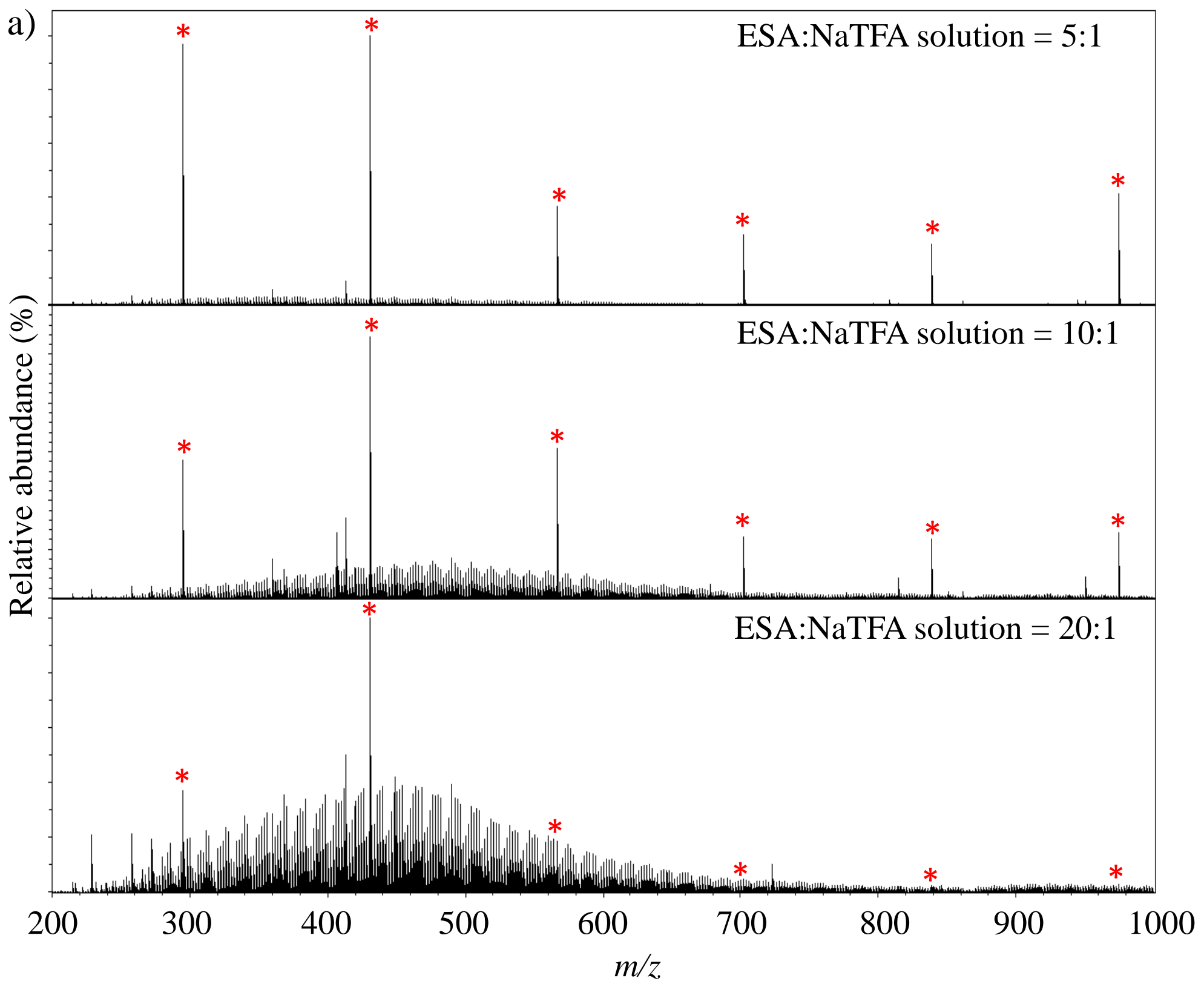


Figure S3. Comparison of broadband spectra obtained by varying the mixing ratio of the ESA crude oil and NaTFA solution from a) (+) ESI FT-ICR MS and b) (-) ESI FT-ICR MS.

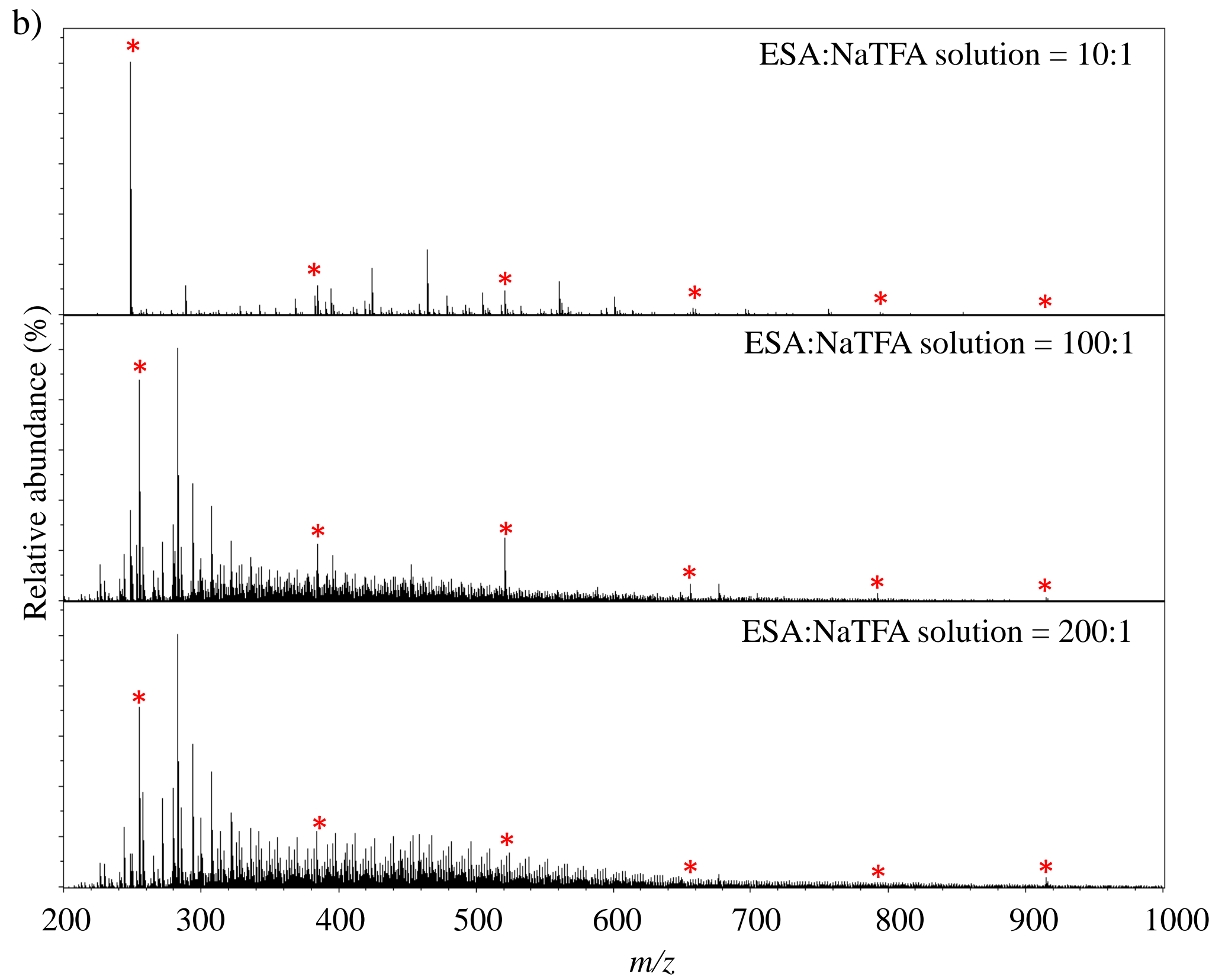


Figure S4. Broadband spectra and mass accuracy plots for the EOC crude oil mixed with NaTFA solution obtained using a) (+) ESI and b) (-) ESI.

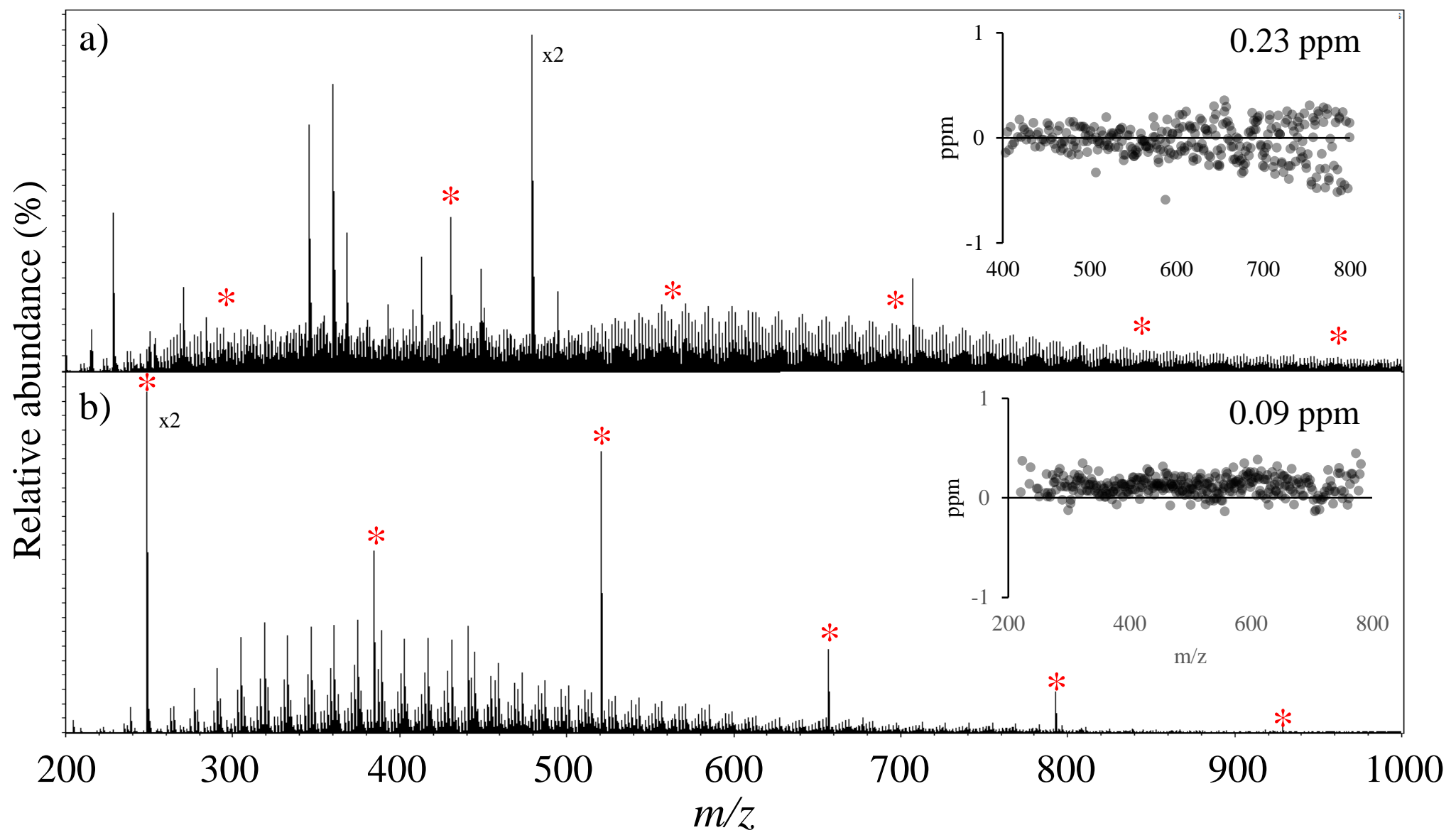


Figure S5. Comparison of broadband spectra obtained by varying the mixing ratio of the SRHA and a) NaTFA or b) arginine solutions obtained using (-) ESI FT-ICR MS

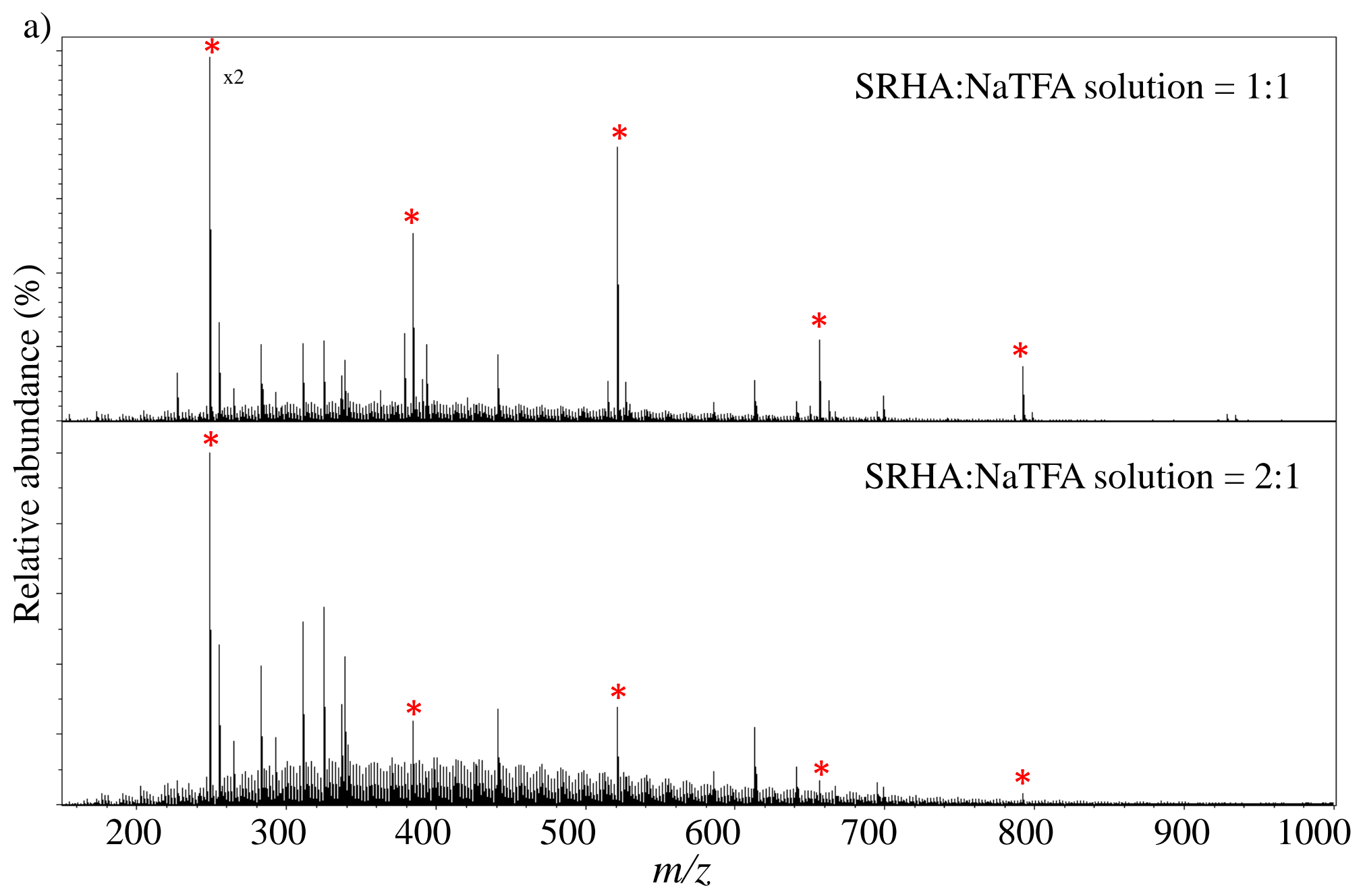


Figure S5. Comparison of broadband spectra obtained by varying the mixing ratio of the SRHA and a) NaTFA or b) arginine solutions obtained using (-) ESI FT-ICR MS

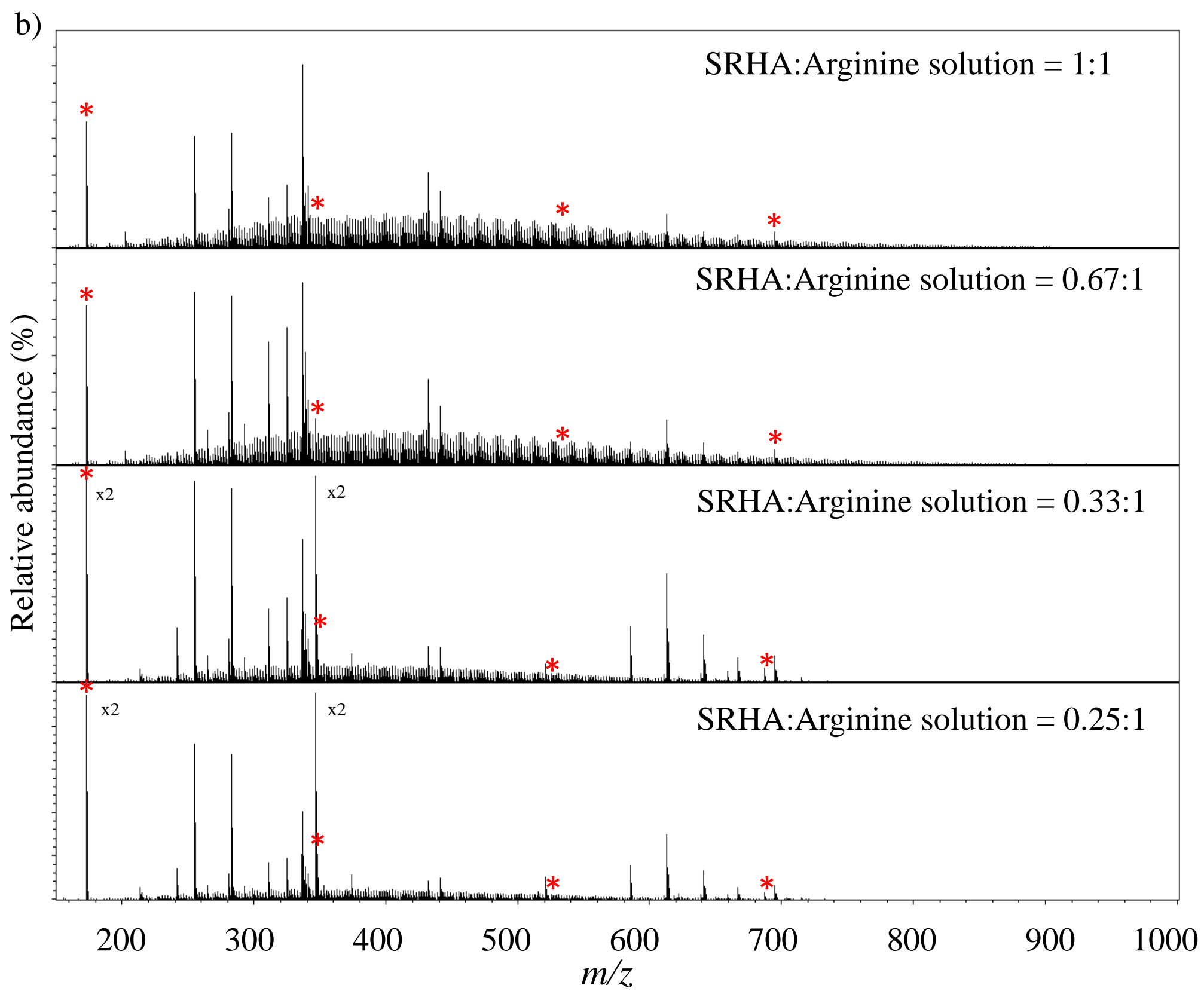


Figure S6. Comparison of broadband spectra obtained upon varying the ratio of the NAP crude oil and RP solution obtained by a) (+) LDI FT-ICR MS and b) (-) LDI FT-ICR MS.

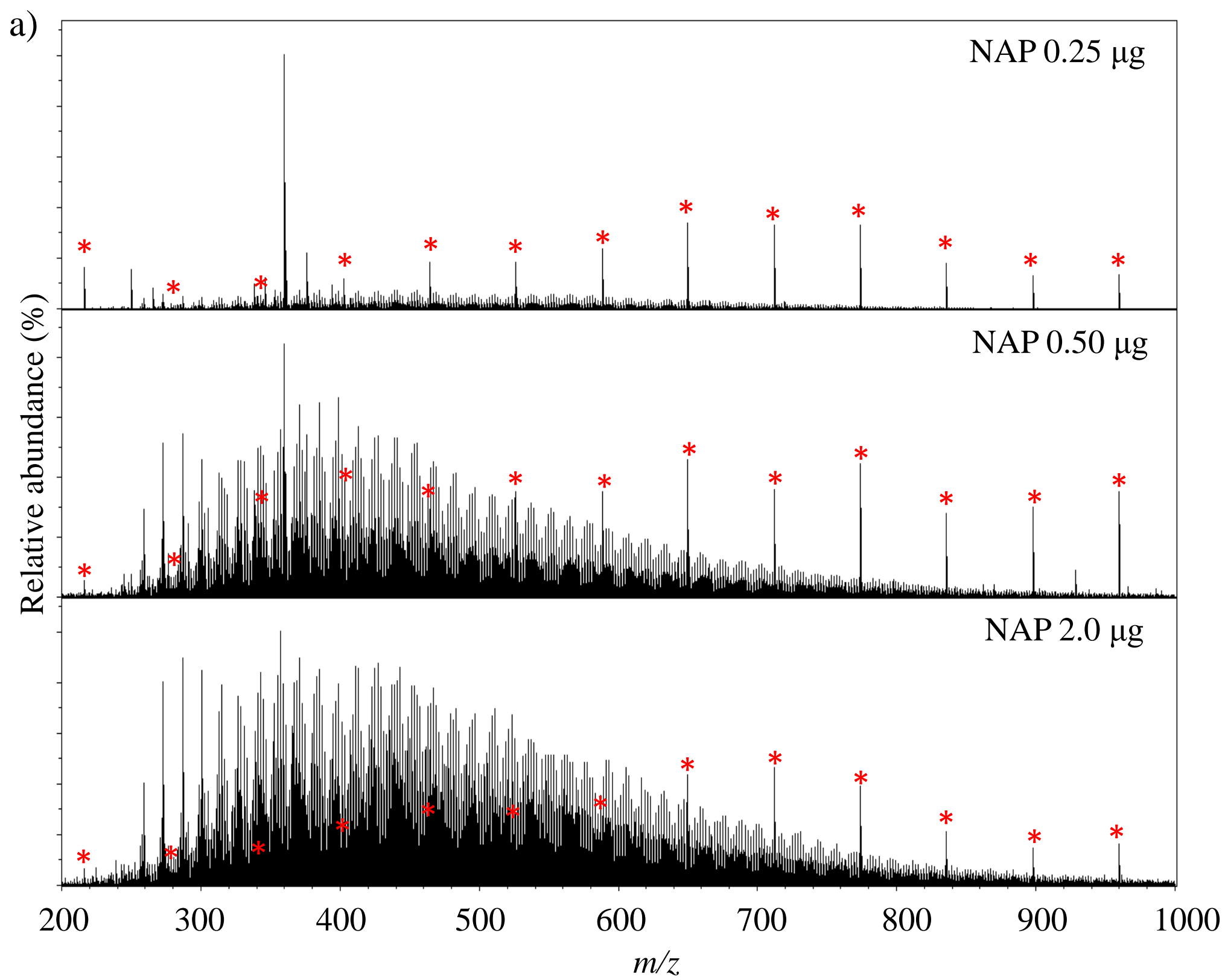


Figure S6. Comparison of broadband spectra obtained upon varying the ratio of the NAP crude oil and RP solution obtained by a) (+) LDI FT-ICR MS and b) (-) LDI FT-ICR MS.

b)

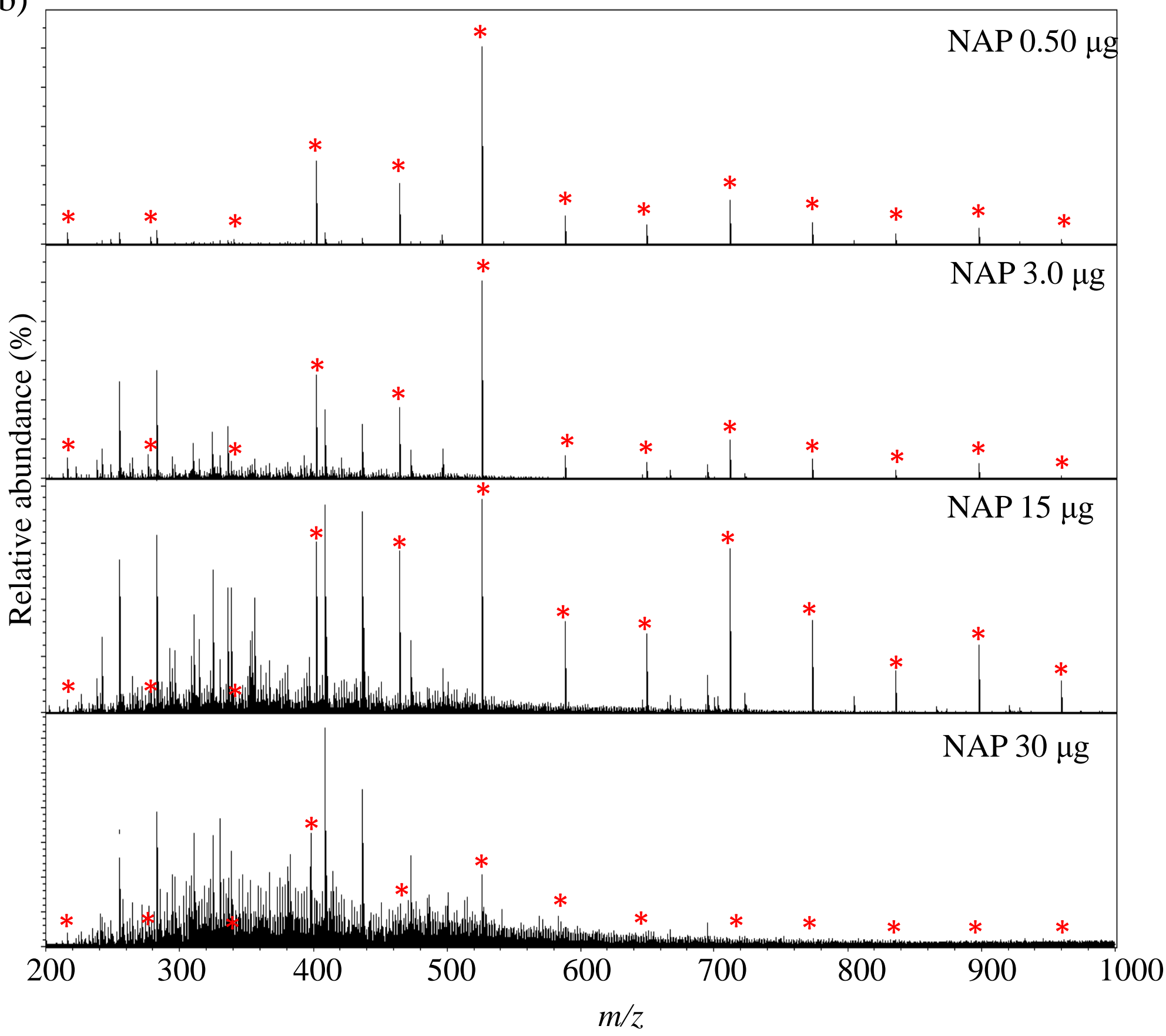


Figure S7. Broadband spectra and mass accuracy plots for the QHD crude oil mixed with RP solution obtained by a) (+) LDI and b) (-) LDI.

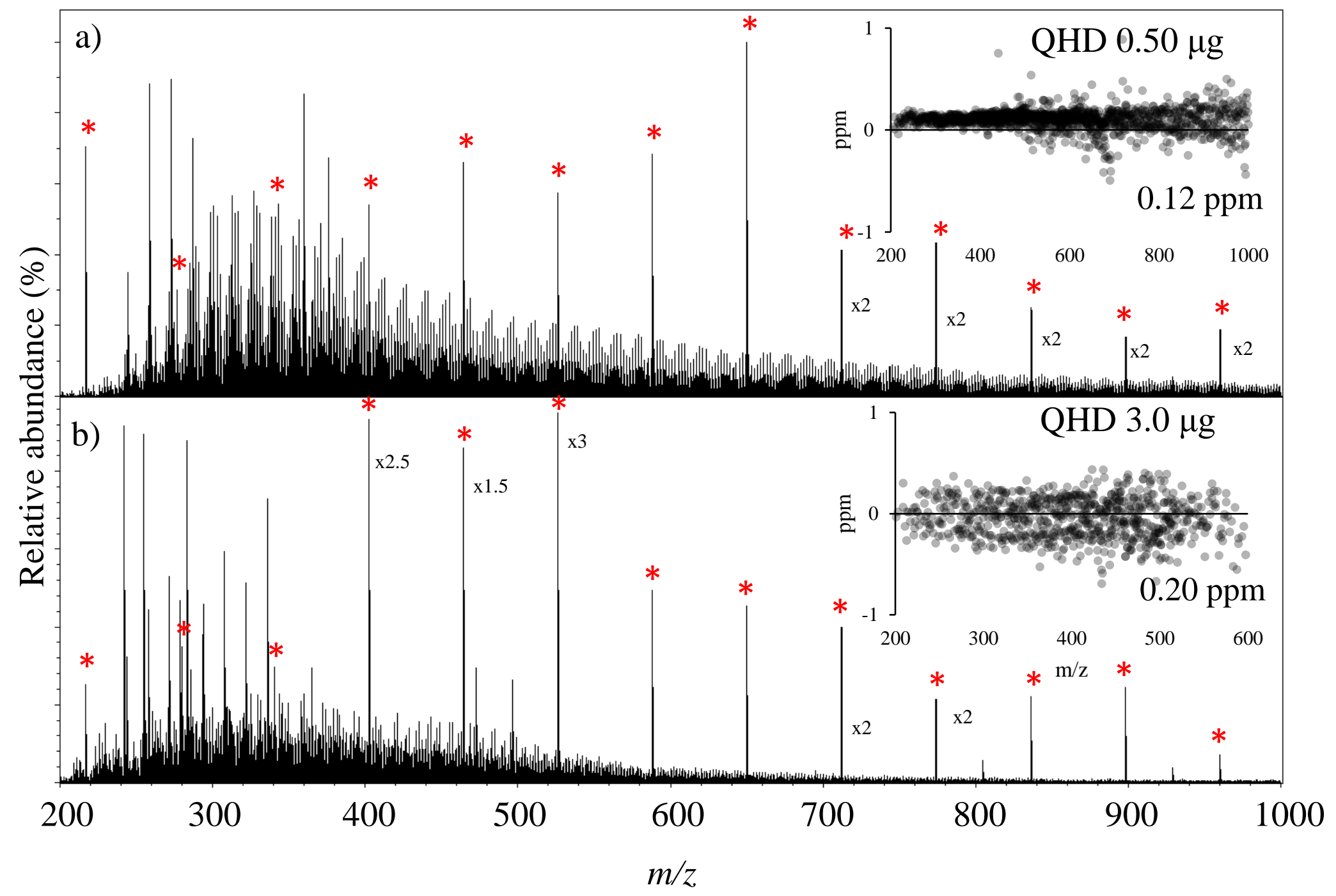


Figure S8. Comparison of broadband spectra obtained by varying the mixing ratio of the SRFA and a) NaTFA or b) RP solutions using (-) LDI FT-ICR MS.

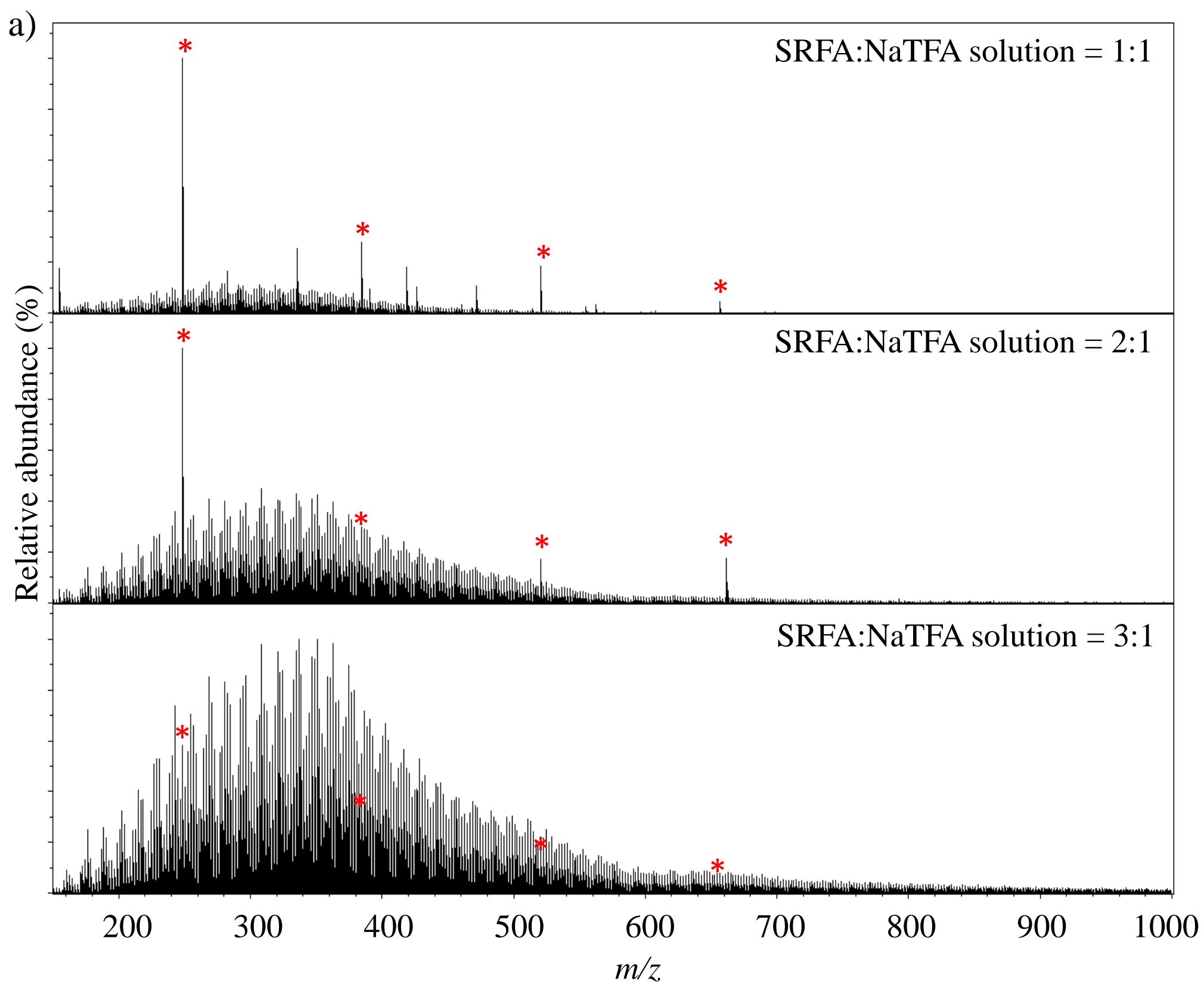


Figure S8. Comparison of broadband spectra obtained by varying the mixing ratio of the SRFA and a) NaTFA or b) RP solutions using (-) LDI FT-ICR MS.

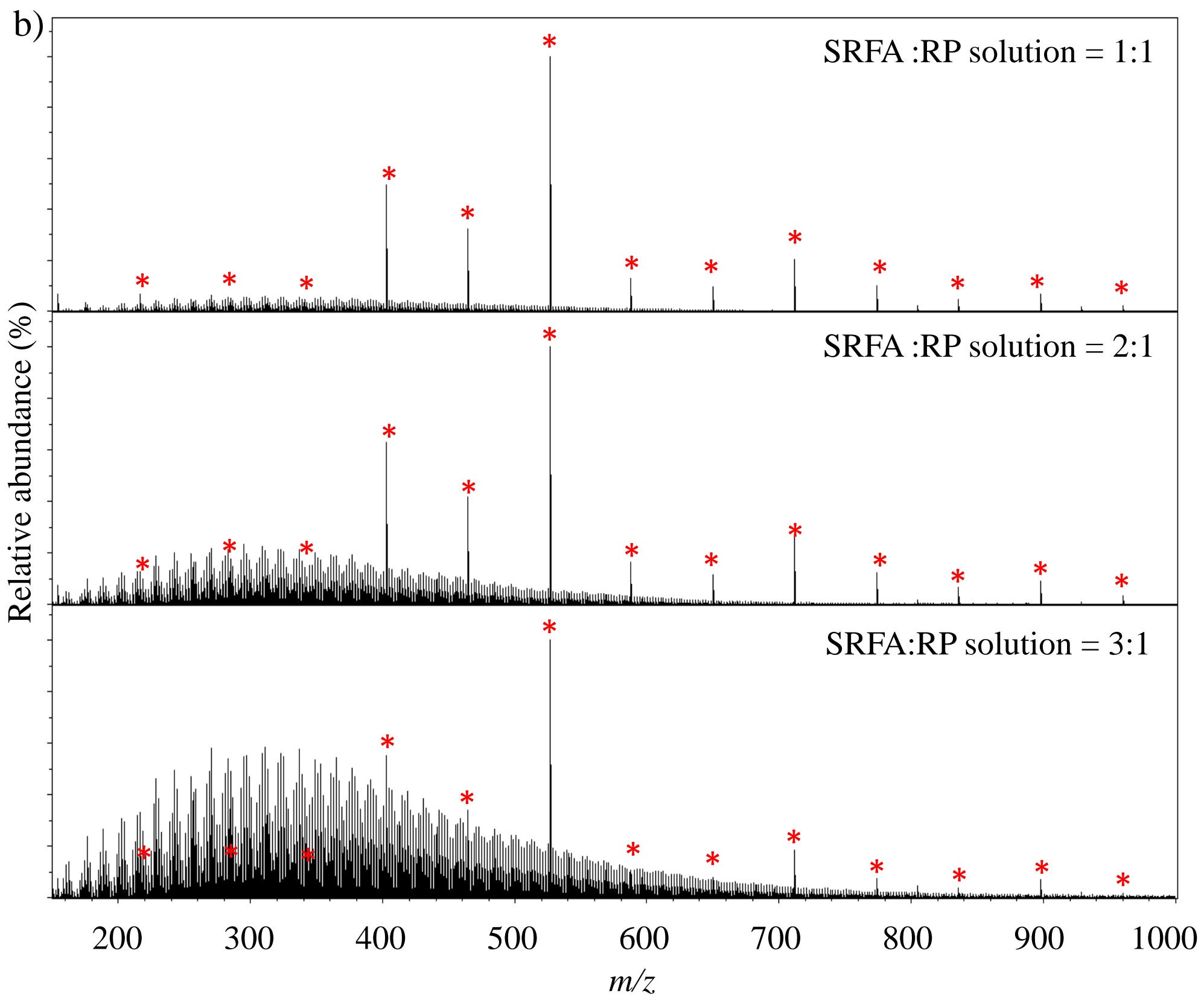


Figure S9. Comparison of broadband spectra obtained by varying the mixing ratio of the SRHA and a) NaTFA or b) RP solutions using (-) LDI FT-ICR MS.

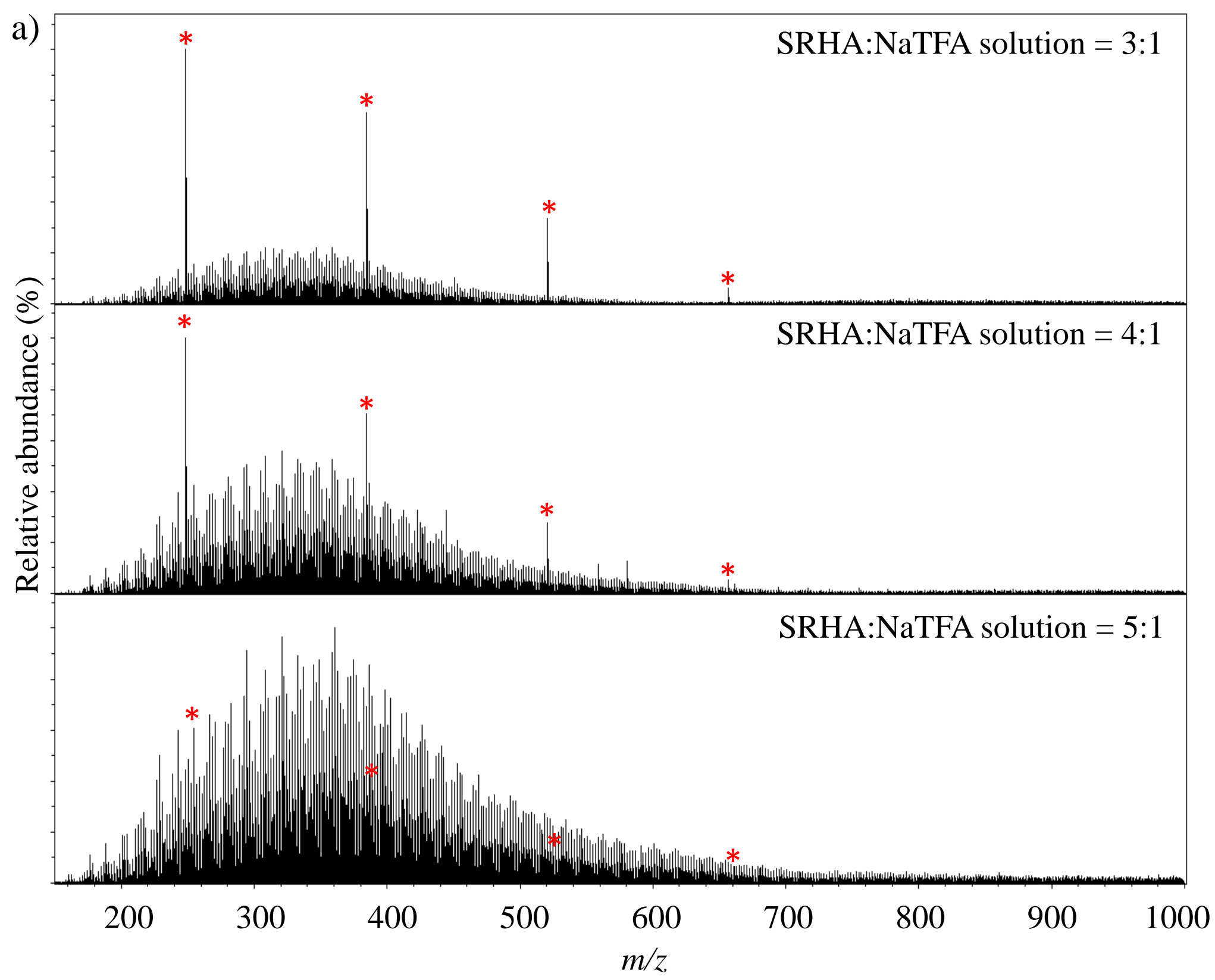


Figure S9. Comparison of broadband spectra obtained by varying the mixing ratio of the SRHA and a) NaTFA or b) RP solutions using (-) LDI FT-ICR MS.

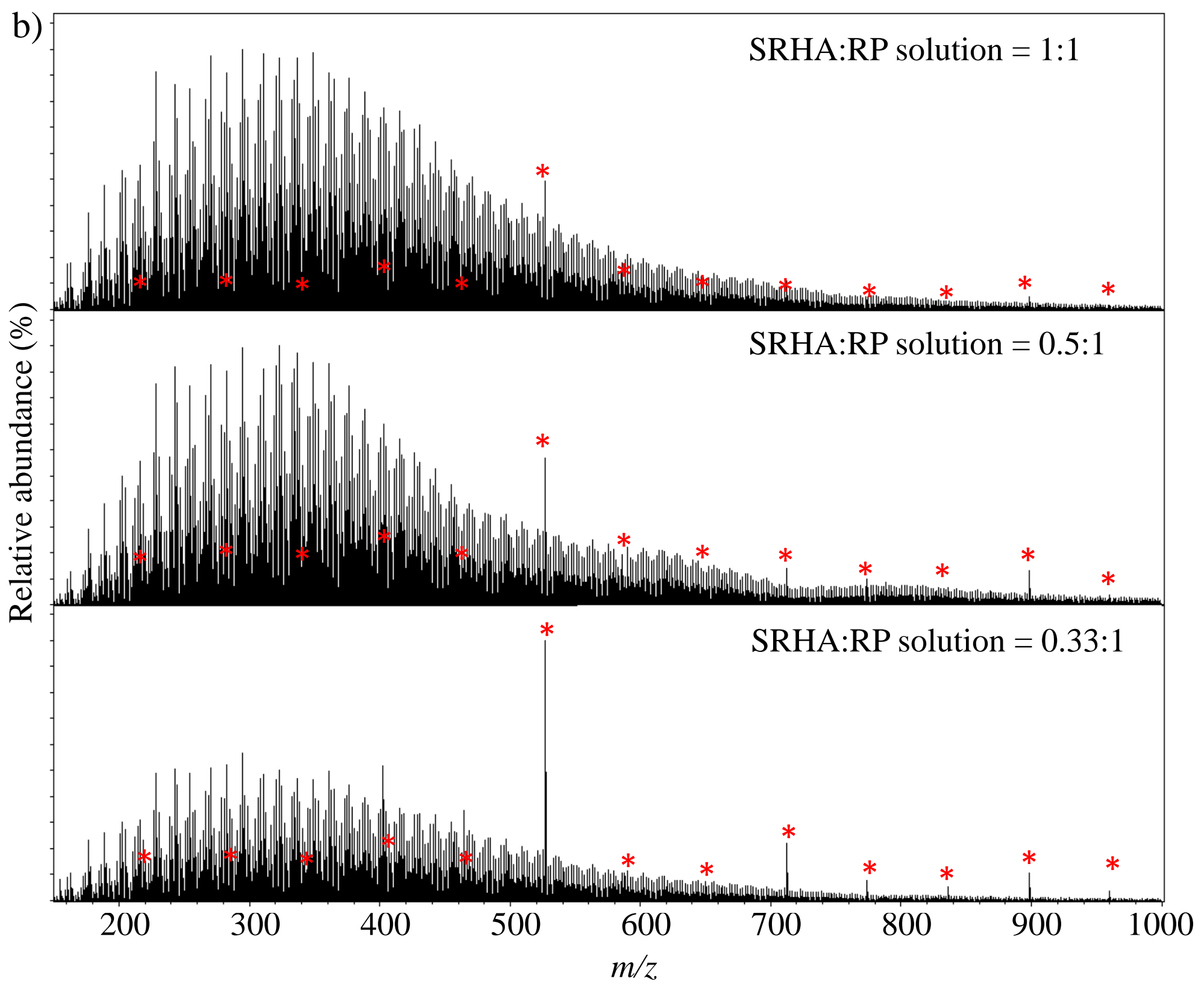


Figure S10. Broadband spectra for the ES mixed with a) arginine solution, b) NaTFA solution, or c) RP solution obtained using (-) LDI FT-ICR MS.

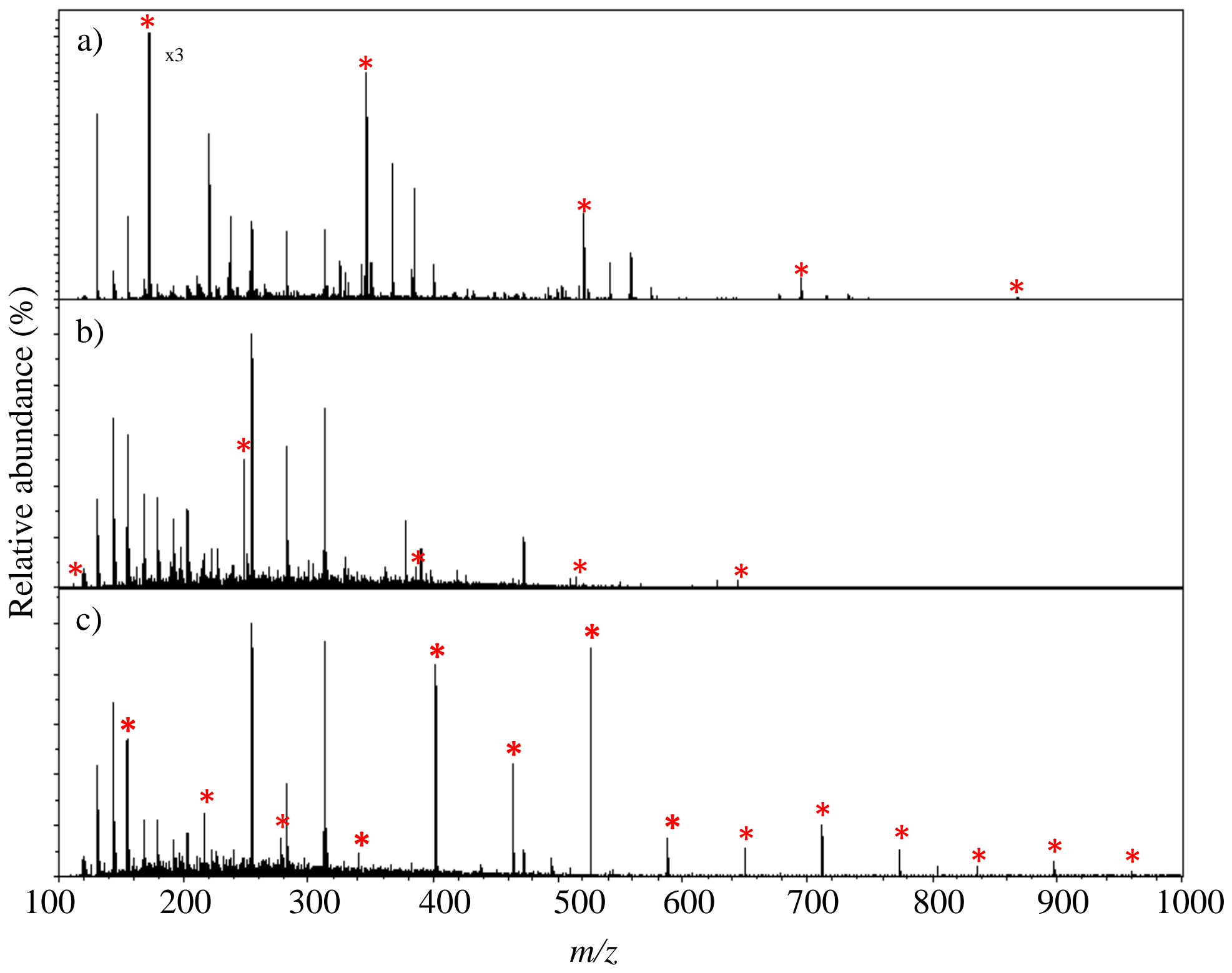

